\title{
BMJ Open ROBOCOP II (ROBOtic assisted versus conventional open partial nephrectomy) randomised, controlled feasibility trial: clinical trial protocol
}

\author{
Karl-Friedrich Kowalewski (D) , ${ }^{1}$ Marie Angela Sidoti Abate, ${ }^{1}$ Manuel Neuberger, ${ }^{1}$ \\ Marietta Kirchner (1) , ${ }^{2}$ Regina Krisam, ${ }^{2}$ Luisa Egen, ${ }^{1}$ Caelan Max Haney, ${ }^{3}$ \\ Fabian Siegel, ${ }^{1}$ Maurice-Stephan Michel, ${ }^{1}$ Patrick Honeck, ${ }^{1}$ Philipp Nuhn, ${ }^{1}$ \\ Niklas Westhoff, ${ }^{1}$ Maximilian Christian Kriegmair ${ }^{1}$
}

To cite: Kowalewski K-F, Sidoti Abate MA, Neuberger M, et al. ROBOCOP II (ROBOtic assisted versus conventional open partial nephrectomy) randomised, controlled feasibility trial: clinical trial protocol. BMJ Open 2021;11:e052087. doi:10.1136/ bmjopen-2021-052087

- Prepublication history for this paper is available online. To view these files, please visit the journal online (http://dx.doi org/10.1136/bmjopen-2021052087).

Received 07 April 2021 Accepted 11 October 2021

Check for updates

(c) Author(s) (or their employer(s)) 2021. Re-use permitted under CC BY-NC. No commercial re-use. See rights and permissions. Published by BMJ.

${ }^{1}$ Department of Urology and Urological Surgery, University Medical Centre Mannheim, Mannheim, Germany Institute of Medical Biometry and Informatics, Heidelberg University, Heidelberg, Germany ${ }^{3}$ Department of Urology, Leipzig University, Leipzig, Germany

Correspondence to Dr Karl-Friedrich Kowalewski; karl-friedrich.kowalewski@ umm.de

\section{ABSTRACT}

Introduction Randomised controlled trials comparing robotic-assisted partial nephrectomy (RAPN) and open PN (OPN) are lacking. Therefore, we aim to report the study protocol and a trial update for a randomised controlled feasibility trial comparing RAPN versus OPN for renal neoplasms.

Methods and analysis The ROBOtic assisted versus conventional Open Partial nephrectomy II trial is designed as a single-centre, randomised, open-label, feasibility trial. Participation will be offered to patients with renal neoplasms and deemed feasible for both, OPN and RAPN. We aim to enrol 50 patients within 15 months using a $1: 1$ allocation ratio. The primary endpoint of the trial is feasibility of recruitment and will be successful if one third of eligible patients agree to participate. Secondary endpoints include perioperative results, health-related quality of life, inflammatory response as well as surgical ergonomics of the operating team. If the primary outcome, feasibility of recruitment, is successful, the secondary results of the trial will be used for planning a confirmative phase III trial.

Ethics and dissemination Ethical approval was obtained from the local institutional review board (Ethik-Kommission II at Heidelberg University: 2020-542N). Results will be made publicly available in peer-reviewed scientific journals and presented at appropriate congresses and social media.

Trial registration number NCT04534998.

\section{INTRODUCTION}

With approximately 99200 new diagnoses in Europe in 2018, renal cell carcinoma (RCC) represents a common malignancy in both men and women. ${ }^{12}$ Surgical resection is the mainstay of curative treatment for RCC. Whenever possible, a nephron sparing approach (partial nephrectomy, PN) should be performed. ${ }^{3}$ PN can either be performed by conventional open PN surgery (OPN) or by a minimally invasive approach, incorporating both robotic-assisted PN (RAPN) and laparoscopic

\section{Strengths and limitations of this study}

- There are currently no available data comparing robotic-assisted partial nephrectomy (PN) and open PN from randomised controlled trials (RCTs).

- The ROBOtic assisted versus conventional Open Partial nephrectomy II trial is essential in order to evaluate whether an independent phase III RCT is realistic and to define effect estimates and meaningful endpoints.

- This trial will help overcome well-known challenges of recruitment in surgical trials and to explore facilitators and barriers for participation for both clinicians and patients.

- Analyses are exploratory rather than confirmative.

PN. International guidelines advise towards choosing an approach that best suits the surgeons' expertise. ${ }^{4}$ This recommendation is the result of a lack of high-level evidence in support of either approach, be it OPN, RAPN or laparoscopic PN. However, with the recent worldwide surge of adoption of RAPN (eg, a reported increase from $0 \%$ in 2006 to $54.4 \%$ in 2014 in the $\mathrm{USA}^{5}$ ), randomised evidence supporting this shift from OPN to RAPN is urgently needed in order to adequately justify this change of treatment. Currently, OPN can be seen as the standard of care in Germany and is performed in around $63 \%$ of cases, while RAPN is performed in approximately $22 \%$ of cases. ${ }^{6}$

Despite being a safe and highly standardised procedure, OPN comes with considerable morbidity such as hernias and flank bulges (in almost $50 \%$ of patients), which can be attributed to the open approach and negatively impacts patients' quality of life (QOL). ${ }^{78}$ Generally, the adoption of minimally invasive surgery to $\mathrm{PN}$ has been 
driven by hopes of tapping into common advantages of minimally invasive surgery. These advantages include but are not limited to less pain, fewer wound infections and a shorter hospital stay thereby resulting in faster recovery. ${ }^{9}$ This is in line with results from the previously published ROBOtic assisted versus conventional Open Partial nephrectomy (ROBOCOP) I trial from our group, a propensity score matched analysis comparing OPN and RAPN. ${ }^{10}$ This study confirmed advantages of RAPN in terms of reduced complications, length of hospital stay as well as higher postoperative glomerular filtration rate (GFR). Similar findings were reported from randomised controlled trials (RCTs) for bladder and prostate cancer comparing robotic versus open surgery. For instance, the RAZOR trial was able to show non-inferiority of roboticassisted radical cystectomy compared with open radical cystectomy in terms of progression-free survival but demonstrated advantages for blood loss, perioperative transfusions and length of hospital stay. ${ }^{11}$ Similar perioperative findings favouring robotic surgery were reported by Yaxley $e t a l^{12}$ comparing robotic-assisted and open radical prostatectomy. Although no clear advantages for either approach were found, robotic-assisted radical prostatectomy is by far the most commonly performed approach for radical prostatectomy.

Looking at kidney cancer, there are currently no published data comparing OPN and RAPN from an RCT. This might be due to the fact that surgical RCTs face unique limitations mainly through the feasibility of randomisation, learning curves, standardisation of procedures as well as patients' and surgeons' equipoise. ${ }^{13}{ }^{14}$ Feasibility trials are helpful as means of specifically addressing these problems as a well-monitored test series with the aim of avoiding pitfalls in later definitive trials. ${ }^{15}$ For example, Stensland et $a l^{16}$ found that $25 \%$ of genitourinary cancer trials are terminated early with poor recruitment being the number one cause with almost $40 \%$. As a matter of fact, the UK Medical Research Council, Consolidated Standards of Reporting Trials ${ }^{17}$ and IDEAL ${ }^{18}$ guidelines explicitly recommend conducting feasibility studies to evaluate key uncertainties before definitive evaluation in large scale RCTs. ${ }^{19}$ Therefore, a feasibility study comparing RAPN and OPN is urgently needed before larger trials can be conducted to answer this question. If feasibility of such an RCT can be proven, a subsequent larger multicentre confirmatory trial is promising and shall be conducted.

Therefore, the primary aim of the ROBOCOP II RCT is to assess feasibility of recruitment in a clinical setting in preparation of a confirmative multicentre RCT, ROBOCOP III.

\section{METHODS AND ANALYSIS}

\section{Trial design and setting}

The ROBOCOP II trial is designed as a single-centre, randomised, open-label feasibility trial to compare RAPN and OPN, performed at the Department of Urology and
Urological Surgery, University Medical Center Mannheim at Heidelberg University, Germany. The trial started on 16 June 2020. We aim to enrol 50 patients that actually undergo surgery within 15 months using a 1:1 allocation ratio. However, because of the ongoing COVID-19 pandemic, the recruitment might take longer than the previously planned 15 months. The estimated recruitment end date is 15 December 2021.The primary outcome will be feasibility of recruitment.

\section{Eligibility criteria}

Inclusion criteria:

- Patient must be at least 18 years old and capable of giving consent.

- Patient must be scheduled for elective PN for localised renal neoplasms.

- Both a robotic assisted or an open approach for surgery must be possible as determined from preoperative imaging by all operating surgeons.

- The patient must be able to understand the goal, consequences and alternatives of participation in the trial.

- Written informed consent.

- Ccurative-intent surgery.

- Abdominal MRI or CT scan.

Exclusion criteria:

- Patients with a solitary kidney.

- Patients belonging to a vulnerable patient group.

- Simultaneous second surgery.

- Emergency intervention, for example, because of bleeding or perforation.

- Multiple kidney tumours.

- Second malignancy that will make PN needless, this does not include secondary malignancies which are under curative treatment or where death from RCC is more likely.

At baseline, patients' biometrical data including age, sex, weight and height will be collected. CT- or MRT scans will be analysed preoperatively in order to record the preoperative aspects and dimensions used for anatomic classification (PADUA),${ }^{20}$ radius exophytic/endophytic nearness anterior/posterior location (RENAL) and Mayo Adhesive Probability (MAP) scores. ${ }^{21} 22$

Healthcare in Germany comprises statutory health insurance, held by about $90 \%$ of population and private health insurance, held by about $10 \%$ of population. ${ }^{23}$ This results in political and social controversies because reimbursement of private health insurance is more lucrative. These two populations might show different healthrelated behaviours. Therefore, healthcare status will also be recorded in order to analyse differences in the decision-making processes among patients with different backgrounds.

\section{Primary outcome}

The primary aim is feasibility of recruitment measured as accrual rate. According to Klabunde $e t a l,{ }^{24}$ recruitment attitude seems to vary among different medical specialties: 
medical oncologists are for instance more likely to participate in recruitment procedures than surgeons.

Generally, the estimated inclusion rate is derived from other multicentre phase III RCTs. Recently, the LEOPARD-2 trial, which compared open pancreaticoduodenectomy with minimally invasive pancreaticoduodenectomy, reported that $42 \%$ of eligible patients eventually participated in the study ${ }^{25}$ Similar data were reported by van der Sluis et $a l,{ }^{26}$ who were able to include almost $50 \%$ of all eligible patients in an RCT comparing open vs minimally invasive esophagectomy. Still, patient accrual represents a barrier in clinical trials. This appears to be the case in urology too, where RCTs with surgical interventions were terminated due to poor patient accrual. ${ }^{27}$ Therefore, ROBOCOP II will be deemed successful if one-third of eligible patients agree to participate since this would enable realisation of a phase III trial with approximately 10 high-volume centres within an acceptable period of time.

\section{Secondary outcomes}

A broad range of secondary endpoints will be collected in order to analyse potential endpoints for a future confirmative multicentre RCT.

QOL (baseline, hospital discharge, 30 and 90 days postoperatively): data regarding patients' QoL will be acquired using the following questionnaires: Kidney Disease Quality of Life Short Form, ${ }^{28}$ Quality of Life Questionnaire C30 (CAT EORTC (Computerised Adaptive Testing - European organisation for Research and Treatment of Cancer) ), ${ }^{29}$ 5-level version of EuroQol-5 Dimension. ${ }^{30}$ Postoperatively: Convalescence and Recovery Evaluation Score. ${ }^{31}$ For patients $\geq 65$ years old, additional questionnaires validated for elderly patients will be administered: Geriatric Depression Scale, ${ }^{32}$ G8 Screening tool, ${ }^{33}$ Selfadministered Comorbidity Questionnaire. ${ }^{34}$

\section{Perioperative parameters}

- Operating time.

- Conversion rate from RAPN to OPN.

- Conversion to radical nephrectomy.

- Intraoperative complications.

- Pleural opening.

- Damage of renal capsule.

- Intraoperative blood loss.

- Blood transfusion.

- Warm ischaemia time.

- Trifecta, defined as no major complication, R0-resection status and warm ischaemia time of $\leq 25 \mathrm{~min}^{35}$

- Surgical peculiarities and/or anomalies.

\section{Surgical ergonomics}

To better understand the advantages and disadvantages of each procedure on the surgeons' part, each operating surgeon will fill out two questionnaires in order to assess ergonomics: the Borg Scale and the NASA-TLX questionnaire. The Borg scale focuses on musculoskeletal pain and will be registered preoperatively and postoperatively ${ }^{36}$;
NASA-TLX (Task Load Index) records psychological and physical load, as well as temporal expenditure, frustration, subjective performance and stress, and will only be administered right after the operation. ${ }^{37}$

\section{Inflammatory markers}

We aim to compare leucocytes, $\mathrm{C}$ reactive protein, interleukin 6 (IL-6), IL 1 beta (IL-1 $\beta$ ), vascular endothelial growth factor and tumour necrosis factor alpha values in both groups.

\section{Need for analgesia}

These recordings will include need for epidural analgesia, opioids, paracetamol, metamizole and other pain medication.

\section{Intraoperative complications}

The "intraoperative adverse incident classification' system by the European Association of Urology will be used to monitor intraoperative complications. ${ }^{38}$

\section{Postoperative complications}

The Comprehensive Complication Index (CCI ${ }^{39}$ which was previously validated for major urological surgery and that is based on the Clavien Dindo classification will be used. ${ }^{40}{ }^{41}$ Since several studies showed lack of standardisation when reporting intraoperative and postoperative complications, ${ }^{42}{ }^{43}$ the latter will be assessed according to the EAU guidelines on reporting and grading complications after urologic surgical procedures. ${ }^{44}$

\section{Kidney function}

For measuring kidney function, the GFR and serum creatinine values will be used. GFR will be calculated by means of the Chronic Kidney Disease Epidemiology Collaboration equation.

\section{Haemoglobin}

Together with transfusion rate, change in haemoglobin values and blood loss will be recorded.

\section{Oncological outcomes}

The histological features of the tumour, and the resection status will be recorded.

\section{Wound healing}

The ASEPSIS (Additional treatment, Serous discharge, Erythema, Purulent exudates, Separation of deep tissues, isolation of bacteria, Stay in hospital) Score will help to assess wound healing. ${ }^{45}$ Moreover, other optical features and wound pain will be registered, together with potential postoperative development of flank bulges.

\section{Case costs}

Case costs will be recorded by means of diagnosis-related groups. For each participant, the nursing costs will be additionally calculated. 


\section{Interventions}

OPN is performed retroperitoneally. A $10-15 \mathrm{~cm}$ flank incision above the 11 th rib is made in order to access the retroperitoneal space. After the exposure of the kidney and the renal hilum, the tumour is identified and excised by clamping the renal artery with a bulldog clamp. Alternatively, the excision is performed in zero-ischaemia technique, depending on tumour diameter, position and surgeon's preference. Resection margins are adapted by secure two-layered renorrhaphy using monofilament sutures. Gerota's Fascia is closed by a polyfilament running suture and the wounds closed in layers.

RAPN is performed transabdominally in flank position with the robotic system (da Vinci Xi, Intuitive Surgical, Sunnyvale, California, USA). A mini-laparotomy, lateral to the umbilicus, allows the placement of the optical trocar and application of the pneumoperitoneum. Subsequently, three trocars for robotic-assisted surgery, as well as an assistant-trocar are placed. After preparation of the renal hilum, the kidney is exposed, and the tumour excised. Resection of the tumour is either performed by clamping of the kidney vessels or in zero-ischaemia technique. Bleeding vessels and the collecting system will be closed by V-Loc sutures and secured by Hem-o-lok. Renorrhaphy is performed by interrupted single sutures using monofilament and secured by Hem-o-lok as well.

Finally, Gerota's Fascia is closed by V-Loc sutures. Retroperitoneal or abdominal drain placement will be omitted for both procedures, as no advantage was shown in existing literature. ${ }^{46} 47$

\section{Recruitment and randomisation}

Any patient fulfilling the eligibility criteria will be offered to take part in the trial at a screening check-up. In case of uncertainty in terms of malignant potential or feasibility of both approaches, patients will be presented to an interdisciplinary team consisting of experienced radiologists and urologists to ensure need for surgery. Patients who decide to participate will receive an information sheet and will sign informed consent. After that, they will be randomised and informed about the surgical approach. Randomisation will be performed as blocked randomisation on a 1:1 allocation basis using a web-based computer algorithm that was developed for urological trials at the Department of Urology and Urological Surgery, University Medical Center Mannheim by the Heinrich-LanzCenter for Digital Health, Medical Faculty Mannheim, Heidelberg University. Block size, which was previously set by an associate that was otherwise not involved in the trial, varies randomly and will be kept confidential until trial completion. Concealment will be ensured by using the aforementioned computer algorithm, which will also generate a unique code for each participant.

\section{Methods against bias}

\section{Selection bias}

All eligible patients will be approached consecutively for inclusion into the trial. The choice of the time point of randomisation is a pragmatic one in order to enable planning of the available operating theatres.

\section{Performance bias}

Only senior attending urologists with a dedicated training in uro-oncological surgery are allowed to participate in the trial. All surgeons are beyond their learning curve as determined from retrospective analysis of perioperative parameters such as trifecta and operating time. ${ }^{48}$ This is in line with previous research that estimated the learning curve for RAPN for experienced surgeons between 16 and 25 cases. $^{49}$ Additionally, the participating surgeons (PN, $\mathrm{PH}$ and MCK) will have to be able to show at least 100 previous PNs and 100 robotic procedures without major adverse events in order to ensure quality of surgical treatment itself. Surgeon will follow standardised techniques as described above.

\section{Detection bias}

All outcomes will be predefined, and most will adhere to strict definitions that do not leave room for interpretation and potential detection bias.

\section{Statistical analysis}

The primary endpoint is the feasibility of recruitment assessed by the accrual rate which is the number of patients fulfilling the eligibility criteria and agreeing to participate in the proposed trial divided by the number of all eligible patients within the recruitment time. Patients that withdraw consent after randomisation and before surgery will be counted as declined participation.

For all secondary outcomes comprehensive data summaries will be given for the whole cohort and stratified by treatment group (if applicable) by means of number of observations and absolute and relative frequencies for categorical and binary variables or mean, SD, median, IQR, Q1, Q3, minimum and maximum. The comparison between the two treatment groups will be based on appropriate statistical tests depending on the distribution of the data; $95 \%$ CIs will be reported. For all secondary outcomes, except for safety relevant endpoints (such as complications) and case costs, comparison of groups will be based on three analyses sets to gain more evidence for the planning of a confirmatory trial: (1) The modified intention-to-treat (mITT) set consists of all randomised patients who underwent surgery. Patients will be analysed in the group they were randomised to (converted patients remain in RAPN group); (2) The per-protocol (PP) set consists of all patients treated PP. Patients with major protocol violation, such as change in treatment group or converted patients, will be excluded; (3) The as-treated (AT) set which considers all patients from the mITT set but patients are analysed in the group they were finally treated in (converted patients in OPN group). Safety relevant endpoints will be analysed in the safety set corresponding to the AT set. For secondary outcomes that are candidate outcomes for a subsequent phase III trial (eg, resection status (R0/R1) and change in kidney function), 
missing values will be imputed to gain more evidence. In case of longitudinal data, (generalised) linear mixed models for repeated measures will be applied to account for missing values. Otherwise, multiple imputation by fully conditional specification will be applied. ${ }^{50}$ Healthcare status will not be analysed by treatment group but for the overall cohort. Descriptive statistics and free text results will be provided. All analyses will be performed using SAS V.9.4 or higher. A statistical analysis plan will be written before data base closure to describe the statistical analysis in more detail. Data analysts will be blinded after assignment to interventions.

\section{Sample size}

Given that this is a feasibility trial, no formal sample size calculation is performed. However, with respect to the primary endpoint, we assume that approximately $35 \%$ of eligible patients that fulfil the inclusion criteria will agree to participate in the proposed trial. This leads to a sample size of $n=25$ patients per group within the planned 15-month recruitment time based on an expected patient number of around 115 patients planned to undergo PN per year. Furthermore, in a subsequent confirmatory trial, candidate outcomes will be evaluated as primary outcome. Considering a $10 \%$ drop-out rate, the width of a $95 \%$ CI for a standardised effect (normal distribution assumed) with $\mathrm{n}=22$ patients per group is $2 \cdot z_{0.975} \cdot \frac{1}{\sqrt{22}}=0.84$, thus illustrating the precision that can be achieved with this trial.

\section{Data management/saving}

Data will be collected using a personalised case report form (CRF) at predefined time intervals that are summed up in table 1 . The data will then be entered in a protected and validated database (https://climedo.de/) approved by the German Federal Office for Information Security and by the Federal Ministry of Health. Only authorised members of the ROBOCOP II study group, comprising study nurses and cooperating doctors, will be permitted to enter, store and access patient data. Climedo holds multiple security certificates (eg, Health Information Trust Alliance, Cloud Security Alliance, Health Insurance Portability and Accountability Act-Protected Health Information) and is encrypted with an SSL-based HTTP protocol (HTTPS). The server's physical location is protected by closed-circuit television and multifactor authentication mechanisms for employees. Every participant will receive a unique subject identification code and a unique patient identification number. Analogue data will be stored and secured at the Department of Urology and Urological Surgery, University Medical Center Mannheim. All data management procedures will be conducted according to written standard operating procedures (SOPs) that guarantee efficient conduct in line with Good Clinical Practice (GCP). At the end of the study, data will be stored for long-term preservation to ensure future reuse.

\section{Study oversight}

Quality assurance will be done in cooperation of monitoring, data management and biostatistics following a riskbased strategy. Risks for patient safety, well-being, patient rights and data validity will be identified and preventive or corrective measures will be taken. Monitoring will be done by the Study Center of the German Society of Surgery and includes clinical on-site visits and will follow SOPs to ensure compliance with the trial protocol, the principles of the Declaration of Helsinki and GCP guidelines as well as data protection and other relevant legal aspects.

The monitor as well as third parties such as representatives of the sponsor or regulatory authorities will be granted access to all trial relevant documents (including CRF, source documents, hospital patient charts and other study files by the investigator. Furthermore, the investigator site file including essential trial related documents like log of staff, delegation log or screening and patient identification $\log$ will be controlled. The monitor will also perform a close-out visit at the trial centre. Risk-based monitoring strategy will be described in a study specific monitoring manual.

On-site monitoring will focus on patient informed consent, screening and feasibility as well as safety according to CCI including control of correct recording and documentation by data verification. Frequency of monitoring visits will be determined depending on recruitment performance.

\section{Funding}

The study will be sponsored by the Dietmar Hopp Stiftung, a non-profit organisation founded in 1995 to enable the implementation of projects in sports, medicine, social issues and education. The Dietmar Hopp Stiftung will not be involved in designing, conducting, analysing, interpreting or writing of the trial report.

\section{Patient and public involvement}

The trial was initiated since more and more patients actively asked for RAPN, despite the lack of high-level evidence. Therefore, in the end of the study, patients will be asked about their personal treatment goals and what is most important for them (oncological control, kidney function, QoL). Moreover patients, urologists and other medical personnel will be interviewed with semistructured interviews to analyse their experience with the study and to detect any potential for improvement.

\section{Limitations}

The results of this trial will be exploratory. However, this trial is essential for a better sample size estimation, for the detection of meaningful endpoints and for the acquisition of first randomised data. Moreover, as in the nature of surgical randomised trials, there might be some unforeseen issues coming up. These can be overcome at the current stage and an amendment will be filed and submitted to the ethics committee if needed. 


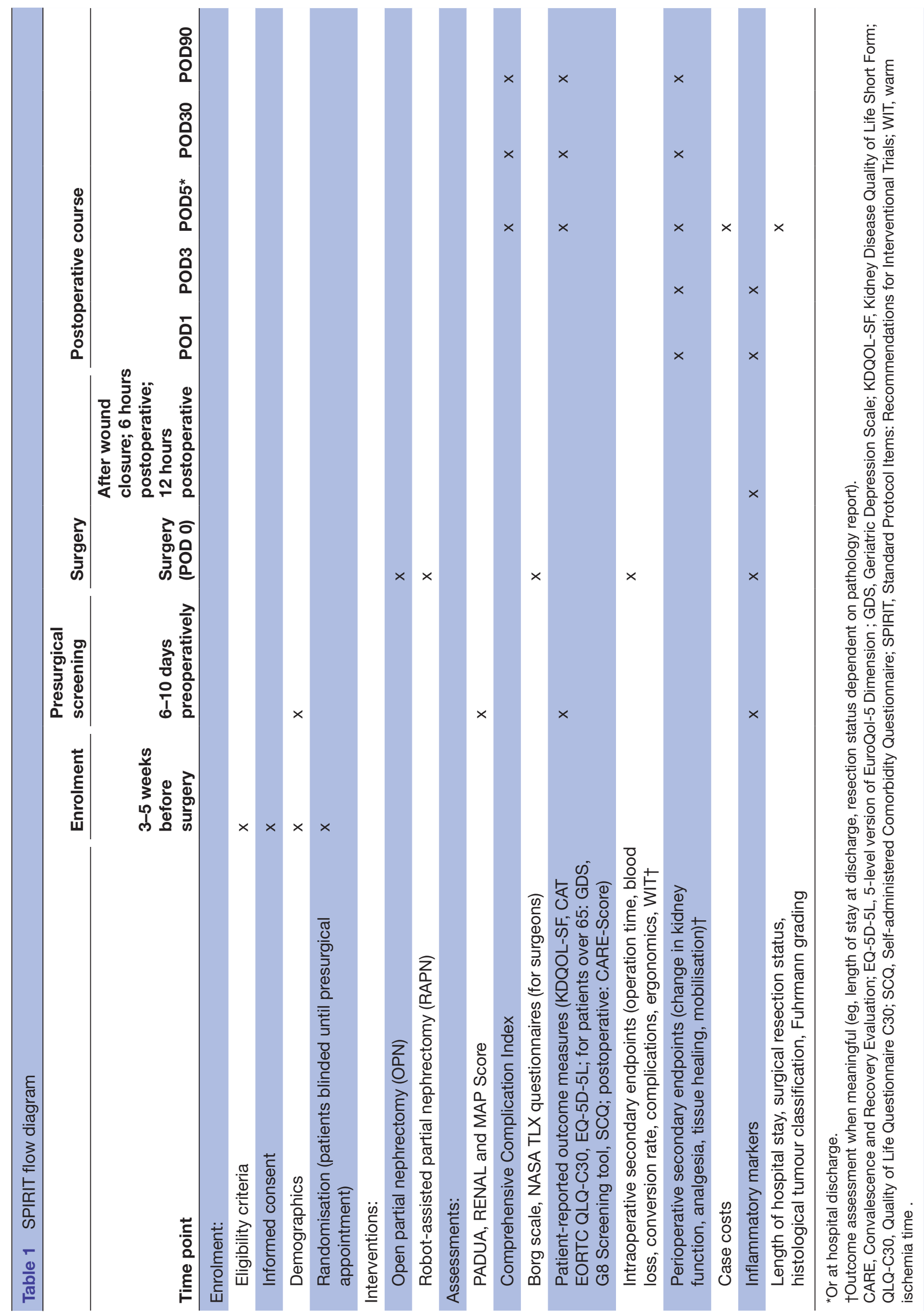

ए

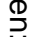

$\stackrel{\vec{F}}{\vec{\rho}}$

흘

$\stackrel{8}{2}$

$\overrightarrow{\vec{\omega}}$

옥

$\frac{0}{8}$

Nิ

$\overrightarrow{\grave{j}}$

잉

ㅇ

w

Z

$\frac{0}{3}$

N

망

응

잉

음

吾

率

긍.

ธิ

웅

을

N

స్心

ए

잉

$\stackrel{\$}{\Phi}$

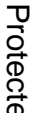




\section{DISCUSSION}

There is currently no published data from RCTs comparing RAPN and OPN. A systematic review and meta-analysis of retrospective studies by Tsai et a $\iota^{51}$ found lower blood loss, fewer transfusions, longer operative times, fewer postoperative complications, lower readmission rate, shorter length of stay and a lower estimated GFR decline for RAPN. Due to low quality of evidence and high heterogeneity, the authors concluded that RCTs are needed. Similar findings were reported in another meta-analysis by Cacciamani et $a \tilde{l}^{2}$ who also compared minimally invasive approaches. Here, RAPN showed better results compared with laparoscopic PN for perioperative parameters (intraoperative complications, ischaemia time, conversion rate), oncological parameters such as positive margins as well as kidney function. ${ }^{52}$ Furthermore, Cacciamani et $a \tilde{l}^{2}$ published a series of meta-analyses focusing on the impact of host factors (eg, tumour complexity, patient comorbidities), hilar control and other factors such as length of hospital stay, readmissions and overall mortality when performing RAPN ${ }^{5354}$ However, even retrospective studies with appropriate statistical methods cannot adjust for unknown group differences and baseline imbalances. This can only be achieved through randomisation.

Although results from retrospective studies seem promising for RAPN, Ramirez et $a l^{55}$ presented negative results for robot assisted surgery in their prospective LACC trial concerning radical hysterectomy. According to their study, laparoscopic or robotic radical hysterectomy was associated with higher cancer recurrence rate and worse overall survival. Consequently, the trial was terminated early for futility. Therefore, randomised evidence is urgently needed to justify the worldwide surge in adopting RAPN to daily practice and to avoid patient harm.

In order to realise such an RCT, a feasibility trial should precede a phase III trial. The importance of conducting feasibility trials has been shown multiple times, with warning examples such as the PREFERE prostatic cancer trial. ${ }^{56}$ The RCT was designed to show non-inferiority of active surveillance, external-beam radiotherapy and brachytherapy by permanent seed implantation to radical prostatectomy with a planned sample size of 7600 patients. However, the trial was stopped early due to poor accrual as only 459 patients agreed to participate within a time period between 2012 and 2016. The realisation of such a study requires the incorporation of all caretakers and patients with their relatives in the process of trial development due to the clinical equipoise and individual beliefs on both sites. ${ }^{57}$ For example, the ORANGE II trial was conducted between January 2010 und July 2014 and recruited 24 patients in 8 study centres before it was terminated due to poor accrual. ${ }^{58}$ Therefore, dedicated staff training including lectures and simulations should be implemented before commencement of the trial in order to optimised workflow and recruitment as done in recent urological feasibility RCTs. ${ }^{59}$ Studies found that telephone reminders, financial incentives, open-trial designs and opt-out procedures are helpful tools to improve accrual. $^{6061}$

As mentioned before, a shift in urological surgery towards the robotic approach must be supported and justified by clinical evidence, bearing in mind not only the costs, but also other significant aspects such as longterm oncological and functional outcomes along with patients' satisfaction and surgeons' performance. In addition to the ROBOCOP II trial, there are currently other RCTs comparing RAPN and OPN being conducted. The CONVERT trial (NCT04011891) is designed as feasibility trial and aims to include a total of 30 patients in Canada. Healthcare systems between different countries differ considerably, as a consequence such a trial is important to enable international trials and to confirm findings among different centres and systems. Furthermore, the phase III OpeRa trial (NCT03849820) is being conducted as a multicentre trial in Germany with industry funding. However, the investigators have reported challenges in recruiting patients. Thus, primary completion rate of the study was postponed from February 2021 to May 2023. Additionally, long-term outcomes were recently added as endpoints and are expected in March 2028. The detailed study protocol including sample size estimation and recalculation is yet to be published. Another trial willing to compare RAPN versus OPN for tumours with a RENAL score higher than seven was recently registered in Egypt (NCT04537247).

In summary, the ROBOCOP II trial is essential in order to evaluate whether an independent phase III RCT is realistic and to provide the basis for effect estimates and meaningful endpoints. Moreover, the trial will help to overcome well-known challenges of recruitment in surgical trials and to explore facilitators and barriers for participation for both, clinicians and patients. Last but not least, it will contribute to the evaluation of which surgical approach is superior, even though all endpoints are exploratory.

Contributors Academic authorship is based on international authorship criteria. Individuals who collaborated in the trial一yet not fulfilling authorship criteria-will be listed as 'cooperators'. All authors contributed to multiple of the following aspects: Study design: K-FK, MASA, MN, CMH, PN, NW and MCK. Data collection: KF-K, MASA and LE. Statistical planning: K-FK, MK, RK and FS. Statistical analysis: MK, RK and FS. Drafting of manuscript: K-FK, MASA, MN, LE, CMH and NW. Patient counselling: K-FK, MASA, NW and MCK. Operative Procedures: PH, PN and MCK. Critical review: M-SM, PH, PN and MCK. Supervision: M-SM, PN and MCK. Thus, all authors contributed significantly to the presented work. In detail, all author met the following criteria: Substantial contributions to the conception or design of the work; or the acquisition, analysis or interpretation of data for the work. Drafting the work or revising it critically for important intellectual content. Final approval of the version to be published. Agreement to be accountable for all aspects of the work in ensuring that questions related to the accuracy or integrity of any part of the work are appropriately investigated and resolved.

Funding This study would not have been possible without funding by the 'Dietmar Hopp Stiftung' (1DH2011061). The authors like to express their gratitude for the support.

Competing interests None declared.

Patient and public involvement Patients and/or the public were not involved in the design, or conduct, or reporting, or dissemination plans of this research.

Patient consent for publication Not applicable. 
Provenance and peer review Not commissioned; externally peer reviewed.

Open access This is an open access article distributed in accordance with the Creative Commons Attribution Non Commercial (CC BY-NC 4.0) license, which permits others to distribute, remix, adapt, build upon this work non-commercially, and license their derivative works on different terms, provided the original work is properly cited, appropriate credit is given, any changes made indicated, and the use is non-commercial. See: http://creativecommons.org/licenses/by-nc/4.0/.

\section{ORCID iDs}

Karl-Friedrich Kowalewski http://orcid.org/0000-0003-2931-6247

Marietta Kirchner http://orcid.org/0000-0003-0294-2483

\section{REFERENCES}

1 Ferlay J, Colombet M, Soerjomataram I, et al. Cancer incidence and mortality patterns in Europe: estimates for 40 countries and 25 major cancers in 2018. Eur J Cancer 2018;103:356-87.

2 Bray F, Ferlay J, Soerjomataram I, et al. Global cancer statistics 2018: GLOBOCAN estimates of incidence and mortality worldwide for 36 cancers in 185 countries. CA Cancer J Clin 2018;68:394-424.

3 Capitanio U, Terrone C, Antonelli A, et al. Nephron-sparing techniques independently decrease the risk of cardiovascular events relative to radical nephrectomy in patients with a $\mathrm{T} 1 \mathrm{a}-\mathrm{T} 1 \mathrm{~b}$ renal mass and normal preoperative renal function. Eur Urol 2015;67:683-9.

4 Ljungberg B, Bensalah K, Canfield S, et al. EAU guidelines on renal cell carcinoma: 2014 update. Eur Urol 2015;67:913-24.

5 Jeong IG, Khandwala YS, Kim JH, et al. Association of roboticassisted vs laparoscopic radical nephrectomy with perioperative outcomes and health care costs, 2003 to 2015. JAMA 2017;318:1561-8.

6 Flegar L, Groeben C, Baunacke M. Der Fokus auf die robotische Nierenteilresektion könnte den konsequenten Organerhalt erschweren: Vergleich populationsbezogener Daten AUS Deutschland und den USA. Der Urologe - Kongressprogramm 2017 2017 https://link.springer.com/article/10.1245/s10434-019-08108-x

7 Chatterjee S, Nam R, Fleshner N, et al. Permanent flank bulge is a consequence of flank incision for radical nephrectomy in one half of patients. Urol Oncol 2004;22:36-9.

8 Kriegmair MC, Younsi N, Hiller K, et al. Single- vs multiplelayer wound closure for flank incisions: results of a prospective, randomised, double-blinded multicentre study. BJU Int 2021;127:6470.

9 Nickel F, Nickel F, Nickel F, et al. Laparoscopic versus open pancreaticoduodenectomy: a systematic review and meta-analysis of randomized controlled trials. Ann Surg 2019;271:54-6.

10 Kowalewksi KF, Müller D, Kirchner M. Robotic-Assisted versus conventional open partial nephrectomy (ROBOCOP): a propensity score-matched analysis of 249 patients. Urol Int 2020;105:490-8.

11 Parekh DJ, Reis IM, Castle EP, et al. Robot-Assisted radical cystectomy versus open radical cystectomy in patients with bladder cancer (razor): an open-label, randomised, phase 3, non-inferiority trial. Lancet 2018;391:2525-36.

12 Yaxley JW, Coughlin GD, Chambers SK, et al. Robot-Assisted laparoscopic prostatectomy versus open radical retropubic prostatectomy: early outcomes from a randomised controlled phase 3 study. Lancet 2016;388:1057-66.

13 Slim K. Limits of evidence-based surgery. World J Surg 2005;29:606-9.

14 McCulloch P, Taylor I, Sasako M, et al. Randomised trials in surgery: problems and possible solutions. BMJ 2002;324:1448-51.

15 Eldridge SM, Lancaster GA, Campbell MJ, et al. Defining feasibility and pilot studies in preparation for randomised controlled trials: development of a conceptual framework. PLoS One 2016;11:e0150205.

16 Stensland KD, McBride R, Wisnivesky JP, et al. Premature termination of genitourinary cancer clinical trials. Journal of Clinical Oncology 2014;32:288.

17 Eldridge SM, Chan CL, Campbell MJ, et al. Consort 2010 statement: extension to randomised pilot and feasibility trials. Pilot Feasibility Stud 2016;2:64.

18 Hirst A, Philippou Y, Blazeby J, et al. No surgical innovation without evaluation: evolution and further development of the ideal framework and recommendations. Ann Surg 2019;269:211-20.

19 Craig P, Dieppe P, Macintyre S, et al. Developing and evaluating complex interventions: the new medical Research Council guidance. BMJ 2008;337:a1655.

20 Ficarra V, Novara G, Secco S, et al. Preoperative aspects and dimensions used for an anatomical (Padua) classification of renal tumours in patients who are candidates for nephron-sparing surgery. Eur Urol 2009;56:786-93.

21 Davidiuk AJ, Parker AS, Thomas CS, et al. Mayo adhesive probability score: an accurate image-based scoring system to predict adherent perinephric fat in partial nephrectomy. Eur Urol 2014;66:1165-71.

22 Kutikov A, Uzzo RG. The R.E.N.A.L. nephrometry score: a comprehensive standardized system for quantitating renal tumor size, location and depth. $J$ Urol 2009;182:844-53.

23 Döring A, Paul F. The German healthcare system. Epma J 2010;1:535-47.

24 Klabunde CN, Keating NL, Potosky AL, et al. A population-based assessment of specialty physician involvement in cancer clinical trials. J Natl Cancer Inst 2011;103:384-97.

25 van Hilst J, de Rooij T, Bosscha K, et al. Laparoscopic versus open pancreatoduodenectomy for pancreatic or periampullary tumours (LEOPARD-2): a multicentre, patient-blinded, randomised controlled phase 2/3 trial. Lancet Gastroenterol Hepatol 2019;4:199-207.

26 van der Sluis PC, van der Horst S, May AM, et al. Robot-Assisted minimally invasive Thoracolaparoscopic esophagectomy versus open transthoracic esophagectomy for resectable esophageal cancer. Ann Surg 2019;269:621-30.

27 Chenam A, Yuh B, Zhumkhawala A, et al. Prospective randomised non-inferiority trial of pelvic drain placement vs no pelvic drain placement after robot-assisted radical prostatectomy. BJU Int 2018;121:357-64.

28 Hays RD, Kallich JD, Mapes DL, et al. Development of the kidney disease quality of life (KDQOL) instrument. Qual Life Res 1994;3:329-38

29 Aaronson NK, Ahmedzai S, Bergman B, et al. The European organization for research and treatment of cancer QLQ-C30: a quality-of-life instrument for use in international clinical trials in oncology. J Natl Cancer Inst 1993;85:365-76.

30 Herdman M, Gudex C, Lloyd A, et al. Development and preliminary testing of the new five-level version of EQ-5D (EQ-5D-5L). Qual Life Res 2011;20:1727-36.

31 Hollenbeck BK, Dunn RL, Wolf JS, et al. Development and validation of the convalescence and recovery evaluation (care) for measuring quality of life after surgery. Qual Life Res 2008;17:915-26.

32 Sheikh J. I, Yesavage JA. Geriatric depression scale (GDS): recent evidence and development of a shorter version. The Journal of Aging and Mental Health 1986;5:165-73.

33 Soubeyran P, Bellera CA, Gregoire F, et al. Validation of a screening test for elderly patients in oncology. Journal of Clinical Oncology 2008;26:20568

34 Sangha O, Stucki G, Liang MH, et al. The self-administered comorbidity questionnaire: a new method to assess comorbidity for clinical and health services research. Arthritis Rheum 2003:49:156-63.

35 Khalifeh A, Autorino R, Hillyer SP, et al. Comparative outcomes and assessment of trifecta in 500 robotic and laparoscopic partial nephrectomy cases: a single surgeon experience. J Urol 2013;189:1236-42.

36 Borg GA. Psychophysical bases of perceived exertion. Med Sci Sports Exerc 1982;14:377???381.

37 Hart SG. Nasa-Task load index (NASA-TLX); 20 years later. Proc Hum Factors Ergon Soc Annu Meet 2006;50:904-8.

38 Biyani CS, Pecanka J, Rouprêt M, et al. Intraoperative adverse incident classification (EAUiaiC) by the European association of urology AD hoc complications guidelines panel. Eur Urol 2020;77:601-10.

39 Kowalewski KF, Müller D, Mühlbauer J, et al. The comprehensive complication index (CCl): proposal of a new reporting standard for complications in major urological surgery. World $\mathrm{J}$ Urol 2021;39:1631-9.

40 Slankamenac K, Graf R, Barkun J, et al. The comprehensive complication index: a novel continuous scale to measure surgical morbidity. Ann Surg 2013;258:1-7.

41 Dindo D, Demartines N, Clavien P-A. Classification of surgical complications: a new proposal with evaluation in a cohort of 6336 patients and results of a survey. Ann Surg 2004;240:205-13.

42 Cacciamani GE, Tafuri A, Iwata A, et al. Quality assessment of intraoperative adverse event reporting during 29227 robotic partial nephrectomies: a systematic review and cumulative analysis. Eur Urol Oncol 2020;3:780-3.

43 Cacciamani GE, Medina LG, Tafuri A, et al. Impact of implementation of standardized criteria in the assessment of complication reporting after robotic partial nephrectomy: a systematic review. Eur Urol Focus 2020;6:513-7.

44 Mitropoulos D, Artibani W, Graefen M, et al. Reporting and grading of complications after urologic surgical procedures: an AD hoc 
EAU guidelines panel assessment and recommendations. Eur Urol 2012;61:341-9.

45 Wilson AP, Treasure T, Sturridge MF, et al. A scoring method (asepsis) for postoperative wound infections for use in clinical trials of antibiotic prophylaxis. Lancet 1986;1:311-2.

46 Kriegmair MC, Mandel P, Krombach P, et al. Drain placement can safely be omitted for open partial nephrectomy: results from a prospective randomized trial. Int J Urol 2016;23:390-4.

47 Kowalewski KF, Hendrie JD, Nickel F, et al. Prophylactic abdominal or retroperitoneal drain placement in major uro-oncological surgery: a systematic review and meta-analysis of comparative studies on radical prostatectomy, cystectomy and partial nephrectomy. World $\mathrm{J}$ Urol 2020;38:1905-17.

48 Harke NN, Mandel P, Witt JH, et al. Are there limits of robotic partial nephrectomy? TRIFECTA outcomes of open and robotic partial nephrectomy for completely endophytic renal tumors. J Surg Oncol 2018;118:206-11.

49 Haseebuddin M, Benway BM, Cabello JM, et al. Robot-Assisted partial nephrectomy: evaluation of learning curve for an experienced renal surgeon. J Endourol 2010;24:57-61.

50 van Buuren S. Multiple imputation of discrete and continuous data by fully conditional specification. Stat Methods Med Res 2007:16:219-42.

51 Tsai S-H, Tseng P-T, Sherer BA, et al. Open versus robotic partial nephrectomy: systematic review and meta-analysis of contemporary studies. Int J Med Robot 2019;15:e1963.

52 Cacciamani GE, Medina LG, Gill T, et al. Impact of surgical factors on robotic partial nephrectomy outcomes: comprehensive systematic review and meta-analysis. J Urol 2018;200:258-74.

53 Cacciamani GE, Gill T, Medina L, et al. Impact of host factors on robotic partial nephrectomy outcomes: comprehensive systematic review and meta-analysis. J Urol 2018;200:716-30.
54 Cacciamani GE, Medina LG, Gill TS, et al. Impact of renal hilar control on outcomes of robotic partial nephrectomy: systematic review and cumulative meta-analysis. Eur Urol Focus 2019;5:619-35.

55 Ramirez PT, Frumovitz M, Pareja R, et al. Phase III randomized trial of laparoscopic or robotic versus abdominal radical hysterectomy in patients with early-stage cervical cancer: LACC trial. Gynecol Oncol 2018;149:245.

56 Wiegel T, Albers P, Bartkowiak D, et al. Results of a randomized trial of treatment modalities in patients with low or early-intermediate risk prostate cancer (PREFERE trial). J Cancer Res Clin Oncol 2021;147:235-42.

57 Donovan JL, de Salis I, Toerien M, et al. The intellectual challenges and emotional consequences of equipoise contributed to the fragility of recruitment in six randomized controlled trials. $J$ Clin Epidemiol 2014;67:912-20.

58 Wong-Lun-Hing EM, van Dam RM, van Breukelen GJP, et al. Randomized clinical trial of open versus laparoscopic left lateral hepatic sectionectomy within an enhanced recovery after surgery programme (orange II study). Br J Surg 2017;104:525-35.

59 Oughton JB, Poad H, Twiddy M, et al. Radical cystectomy (bladder removal) against intravesical BCG immunotherapy for high-risk non-muscle invasive bladder cancer (BRAVO): a protocol for a randomised controlled feasibility study. BMJ Open 2017;7:e017913.

60 Elliott D, Husbands S, Hamdy FC, et al. Understanding and improving recruitment to randomised controlled trials: qualitative research approaches. Eur Urol 2017;72:789-98.

61 Treweek S, Lockhart P, Pitkethly M, et al. Methods to improve recruitment to randomised controlled trials: cochrane systematic review and meta-analysis. BMJ Open 2013;3:e002360. 